\title{
FRAÇÕES DA MATERIA ORGÂNICA, INDICE DE MANEJO DO CARBONO E ATRIBUTOS FÍSICOS DE UM LATOSSOLO VERMELHO SOB DIFERENTES SISTEMAS DE USO
}

\section{Fractions of organic matter, carbon management index and physical attributes of a red oxisol under different use systems}

\author{
Nilson Marcos Balin ${ }^{1}$, Ana Regina Dahlem Ziech², Jaqueline Pereira Machado de Oliveira ${ }^{3}$, Vitor Cauduro \\ Girardello ${ }^{4}$, Lizete Stumpf ${ }^{5}$, Paulo Cesar Conceição ${ }^{6 *}$
}

\author{
${ }^{1}$ Engenheiro Florestal, extensionista do Instituto Paranaense de Assistência Técnica e Extensão Rural, Dois Vizinhos, \\ PR, Brasil; nilson.eng-ab@hotmail.com \\ 2 Professora, Universidade Tecnológica Federal do Paraná- Campus Santa Helena (UTFPR-SH); Santa Helena - PR, Brasil, e-mail: \\ anaziech@utfpr.edu.br \\ ${ }^{3}$ Pós-doutoranda PNPD, Pós-Graduação em Agronomia Universidade Tecnológica Federal do Paraná (UTFPR-PB); e-mail \\ jaqueline.oliveira@unoesc.edu.br \\ 4 Pós-doutorando PNPD, Pós-Graduação em Agronomia Universidade Tecnológica Federal do Paraná (UTFPR-PB), e-mail: \\ vitorgirardello@santoangelo.uri.br \\ 5 Pós-doutoranda PNPD-CAPES, Pós-Graduação em Agronomia Universidade Tecnológica Federal do Paraná (UTFPR-PB); e- \\ mail: zete.stumpf@gmail.com \\ ${ }^{6}$ Professor, Universidade Tecnológica Federal do Paraná- Campus Dois Vizinhos, Coordenação em Agronomia; Dois Vizinhos - \\ PR, Brasil; (* autor para correspondência), e-mail: paulocesar@utfpr.edu.br.
}

Artigo enviado em 08/06/2017, aceito em 10/07/2017 e publicado em 29/09/2017.

Resumo - O presente estudo teve como objetivo analisar as frações da matéria orgânica, o índice de manejo do carbono e os atributos físicos de um Latossolo Vermelho sob diferentes sistemas de uso. Quatro áreas distintas de uso do solo foram avaliadas: Lavoura de culturas anuais sob sistema plantio direto há mais de 5 anos (LAV-PD); Pastagem com grama estrela (PAST); Plantio florestal de eucaliptos com 10 anos de idade (EUC); Floresta Ombrófila Mista em estagio médio de regeneração (MATA). Amostras de solo foram coletadas nas camadas de 0,00-0,05, 0,05-0,10, 0,100,20 e 0,20-0,40 m para a determinação do teor de carbono orgânico total (COT), do carbono orgânico particulado (COP), do carbono orgânico associado aos minerais (CAM), do diâmetro médio geométrico de agregados (DMG), da densidade do solo (Ds), da macroporosidade (Ma), da microporosidade (Mi) e da porosidade total (Pt). O estudo mostrou a importância de se realizar avaliações quali-quantitativas do teor de carbono do solo (COT, COP e CAM), no intuito de se avaliar de uma forma mais precisa a contribuição dos sistemas de uso sobre a dinâmica do carbono no ambiente e nos atributos físicos do solo. O tratamento MATA mostrou-se ser o sistema mais equilibrado, considerando todas as camadas de solo avaliadas, pois apresentou a maior estabilidade dos agregados, menor Ds e maior percentual de Pt e Ma em relação aos demais tratamentos.

Palavras chave - carbono orgânico total, carbono orgânico associado aos minerais, carbono orgânico particulado, agregação, densidade do solo, macroporosidade.

Abstract - The present study aimed to analyze fractions of organic matter, carbon management index and the physical attributes of a Red Latossol under different use systems. Four distinct areas of land use had been selected: No-tillage annual crops 5 years old; Area with pasture ; forest area with eucalyptus plantation 10 years old; MixedRain Forest in average stage of regeneration. Soil samples were collected in layers of $0.00-0.05,0.05-0.10,0.10-0.20$ and $0.20-0.40 \mathrm{~m}$ for determination of total organic carbon, particulate organic carbon, organic carbon associated with minerals, the geometric mean diameter of aggregates, bulk density, the macroporosity, the microporosity and total porosity and . The study showed the importance of conducting evaluations quali-quantitative soil carbon content, in order to assess more precisely the contribution of systems for use on the carbon dynamics in the environment and in the physical attributes of the soil. The treatmentForest proved to be the most balanced, considering all the layers of soil are evaluated, as presented greater stability of aggregates, lower bulk density and higher percentage of total porosity and macroporosity compared to other treatments.

Key words - total organic carbon, organic carbon associated with minerals, particulate organic carbon, aggregates, bunk density, macroporosity. 


\section{INTRODUÇÃO}

Em ecossistemas naturais, o solo apresenta um conteúdo de carbono orgânico estabilizado, que reflete as condições ambientais determinadas pelo clima, vegetação, topografia, e, por características do próprio solo. Contudo, esse estado é alterado quando o solo passa a ser utilizado para fins agrícolas (BRAIDA; REICHERT, 2014). Quando o sistema solo perde carbono, devido a redução dos teores de matéria orgânica do solo, gera uma diminuição de sua qualidade (LAL, 2015), interferindo na sua agregação (SOUZA et al., 2016; FONTE et al., 2014), porosidade e densidade (ROSSETTI; CENTURION, 2015).

As formas de uso e manejo do solo são responsáveis pela entrada de carbono no sistema, bem como pela sua saída do solo para atmosfera. Nos sistemas agrícolas, essa entrada/saída de carbono é muito influenciada pelo preparo do solo, espécies utilizadas, rotações de culturas, adubação e, principalmente, pelo manejo dos resíduos das culturas (COSER et al., 2016; CAMPOS et al., 2011).

De acordo com Dick et al. (2009), o principal processo de adição de material orgânico ao solo tem a planta como componente ativo, consequentemente, o estoque de matéria orgânica do solo é resultado dos processos de adição e perda de material orgânico, onde as plantas e os microrganismos desempenham papel fundamental nessa dinâmica. O carbono pode acumular em frações lábeis ou estáveis da matéria orgânica no solo (MOS), o que pode ter implicações na duração do seu efeito sobre as propriedades do solo (REIS et al., 2016; AMORIM, 2016).

Por conta desta importância, diferentes frações da matéria orgânica do solo, como o carbono orgânico particulado (COP) e o carbono associado aos minerais (CAM), vêm sendo utilizados como indicadores de qualidade do solo, por serem mais sensíveis às alterações de manejo do solo do que o carbono orgânico total (COT), conforme observado em Faccin et al. (2017), Kunde et al. (2016), Leal et al. (2016), Salton et al. (2012) e Loss et al. (2011).

O COP contribui com cerca de 3 a $20 \%$ do COT do solo e é composto, principalmente, por restos vegetais em vários estágios de alteração, apresentando partículas maiores que $0,053 \mathrm{~mm}$ (CONCEIÇÃO, 2006). Esta fração pode apresentar-se em elevadas quantidades na camada mais superficial de solos sob plantio direto, por conta do maior aporte de resíduos em sua superfície. Por outro lado, o COP pode apresentar-se em maiores quantidades nas camadas inferiores a $0,05 \mathrm{~m}$ em solos sob plantio convencional, devido à incorporação dos resíduos em profundidade, conforme observado por Amorin (2016).

O CAM, responsável pelas associações com os argilominerais do solo, é o material orgânico em estágio mais avançado de decomposição, apresentando partículas $<0,053 \mathrm{~mm}$ (CONCEIÇÃO, 2006) e em geral está fração mostra-se menos sensível ao manejo do solo, principalmente no curto prazo, conforme observado por Kunde et al. (2016) e Ensinas et al. (2016).

Adicionalmente, ao considerar a fração lábil (COP) e não lábil (CAM) da matéria orgânica do solo, muitos autores tem adotado o índice de manejo de carbono (IMC) para comparar sistemas de manejo quanto à capacidade de melhorar a qualidade do solo, enfatizando que, quanto maior o valor de IMC, maior é a qualidade do manejo (LEAL et al., 2016; SOUZA et al., 2016; REIS et al., 2016). De acordo com Conceição et al. (2014) o IMC visa avaliar de forma conjunta o efeito dos sistemas de manejos na quantidade e na qualidade (labilidade) da matéria orgânica do solo.

Uma forma de avaliar ou determinar a relação existente entre as práticas de manejo e a qualidade do solo é através do monitoramento de seus atributos (LAL, 2015), principalmente àqueles ligados à sua condição física, pois são os que apresentam maior vulnerabilidade às mudanças no sistema de manejo (CUNHA et al., 2012).

De acordo com Brady e Weill (2013) práticas específicas para reduzir as perdas de carbono em ecossistemas agrícolas incluem um melhor controle da erosão do solo e o uso de cultivos conservacionistas, como o plantio direto na palha. Consequentemente, os solos sob estes manejos tendem a apresentar, ao longo do tempo, uma melhor qualidade física, química e biológica, garantindo a produtividade das culturas e a conservação do ambiente.

Neste sentido, o objetivo do trabalho foi avaliar o efeito dos diferentes sistemas de uso nos teores de COT, COP, CAM, no IMC e nos atributos físicos de um Latossolo Vermelho.

\section{MATERIAL E MÉTODOS}

O estudo foi conduzido na Universidade Tecnológica Federal do Paraná - Campus Dois Vizinhos, com localização de latitude $-25^{\circ} 41^{\prime} 50^{\prime \prime}$, longitude de $-53^{\circ} 05^{\prime} 56^{\prime}$ 'W e altitude média de 530 metros. O solo é classificado como um Latossolo Vermelho (EMBRAPA, 2013), com textura muito argilosa $\left(224 \mathrm{~g} \mathrm{~kg}^{-1}\right.$ de silte; $773 \mathrm{~g} \mathrm{~kg}^{-1}$ de argila e $30 \mathrm{~g}$ $\mathrm{kg}^{-1}$ de areia). O clima, segundo a classificação de Koppen (ALVARES et al., 2013), é do tipo Cfa subtropical úmido mesotérmico com verão quente, sem estação seca definida. A temperatura média do mês mais frio é inferior a $18^{\circ} \mathrm{C}$ e o mês mais quente é acima de $22^{\circ} \mathrm{C}$. A formação vegetal original é caracterizada como floresta Ombrófila mista em estágio médio de regeneração.

Amostras de solo foram coletadas $2012 \mathrm{em}$ quatro áreas com diferentes sistemas de uso do solo:

a) Área com culturas anuais manejado sob o sistema plantio direto há mais de 5 anos (LAV-PD): cultivado 
com milho no verão e aveia no inverno, com manejo totalmente mecanizado;

b) Área com pastagem (PAST): formado pela espécie grama-estrela (Cynodonnlemfuensis) há 8 anos, a qual é utilizada para repouso do gado no período noturno. A pastagem se encontra em níveis estáveis de conservação, devido ao seu manejo adequado e ao baixo nível de pisoteio e pastejo no período diurno.

c) Área com plantio florestal de eucaliptos (EUC): formado pela espécie de Eucalyptusurophylla, com 10 anos de idade. O espaçamento é de 2,0 × 2,5 m, com 2000 árvores por hectare. Nesse plantio foram realizados tratos silviculturais como coroamento, desrama aos dois e quatro anos de idade, porém, não foi realizado nenhum tipo de desbaste;

d) Área com floresta Ombrófila Mista (MATA) em estágio médio de regeneração.

Em cada tratamento, foram coletadas 18 amostras de solo com estrutura não preservada, nas camadas de $0,00-0,05,0,05-0,10,0,10-0,20$ e $0,20-0,40$ $\mathrm{m}$, para a determinação do teor de carbono orgânico total, segundo a metodologia de Yeomans e Bremner (1988), e do fracionamento físico granulométrico, segundo a metodologia de Cambardella e Elliott (1992). O material retido na peneira consiste no COP $(>0,053$ $\mathrm{mm})$ e o material que passou pela peneira de $0,053 \mathrm{~mm}$, consiste no CAM, o qual foi obtido por diferença entre o COT e COP.

O cálculo do IMC e de seus componentes foi realizado conforme Blair et al. (1995), com as adaptações de Diekow et al. (2005), em que o COP corresponde à fração lábil da MOS e o CAM corresponde à fração não lábil, segundo a expressão $\mathrm{IMC}=\mathrm{IEC} \times \mathrm{ILC} \times 100$, em que: IEC, é o estoque de COT do tratamento/estoque de COT da referência; ILC é a labilidade do C no tratamento/ labilidade do C na referência; $\mathrm{L}$ é o estoque de $\mathrm{C}$ orgânico lábil/estoque de $\mathrm{C}$ orgânico não lábil. O IMC, portanto, vem a ser uma medida relativa das alterações provocadas pelo manejo ao solo, comparando-o com uma situação considerada original ou ideal, chamada de referência, que neste estudo, foi considerada a área com floresta Ombrófila Mista.

Adicionalmente, em cada tratamento, foram coletadas 6 amostras de solo com estrutura preservada nas camadas de $0,0-0,05,0,05-0,10,0,10-0,20$ e 0,20 $0,40 \mathrm{~m}$ para a determinação da estabilidade de agregados em água, segundo Carpernedo e Mielniczuk (1990). Para interpretação dos resultados dos agregados utilizou-se o parâmetro de diâmetro médio geométrico (DMG). Também foram coletadas, em cada tratamento, 6 amostras de solo com estrutura preservada nas camadas de $0,0-0,05,0,05-0,10,0,10-0,20$ e $0,20-0,40 \mathrm{~m}$ para a determinação da densidade do solo (Ds), da porosidade total (PT), da macroporosidade (Ma) e da microporosidade (Mi), segundo Donagema et al. (2011).

Os resultados obtidos foram analisados pelo programa estatístico Assistat Beta (SILVA;
AZEVEDO, 2009), realizando o teste de comparação de médias (Tukey, $\mathrm{p}<0,05$ de probabilidade).

\section{RESULTADOS E DISCUSSÃO}

Na tabela 1 se observa que os diferentes sistemas de uso do solo apresentaram diferenças significativas quanto ao teor de COT, COP e CAM somente na camada $0,00-0,05 \mathrm{~m}$. O tratamento MATA apresentou os menores teores de COT $\left(6,62 \mathrm{Mg} \mathrm{ha}^{-1}\right)$ em relação aos tratamentos EUC $\left(10,33 \mathrm{Mg} \mathrm{ha}^{-1}\right)$ e LAV-PD (8,72 $\left.\mathrm{Mg} \mathrm{ha}^{-1}\right)$, não diferindo do tratamento PAST $\left(7,80 \mathrm{Mg} \mathrm{ha}{ }^{-1}\right)$. Este resultado deve-se, possivelmente, a maior atividade biológica e consequentemente a maior ciclagem de resíduos que ocorrem em um sistema natural, principalmente nas camadas mais superficiais do solo, conforme exposto por Balota (2017).

O maior teor do COT das áreas de EUC e LAV-PD deve-se, possivelmente, ao maior aporte de resíduos culturais depositados em superfície. Todavia, ao se avaliar qualitativamente as frações da MOS se observou que a fração de COP do tratamento MATA representou $73 \%$ do teor de COT na camada de 0,00 $0,05 \mathrm{~m}$, enquanto que o COP do tratamento EUC representou apenas $14 \%$, compartimento menor inclusive em relação aos tratamentos LAV-PD (30\%) e PAST (32\%) (Tabela 1). Este resultado diverge dos autores Santos et al. (2013); Lima et al (2008) e Mendham et al.(2004), os quais observaram maiores valores de COT e COP no solo sob cultivo de eucalipto, atribuindo esse resultado somente à maior deposição de resíduos na superfície do solo. Os motivos que levaram a resultados distintos, em relação ao teor de COP dos autores citados acima e o apresentado neste trabalho não são evidentes, mas podem incluir diferenças na idade da floresta por ocasião da coleta das amostras, avaliações desta fração em camadas menores ou maiores que $0,05 \mathrm{~m}$, manejo anterior à implantação do eucalipto, clima, dentre outros fatores.

Entre os tratamentos LAV-PD, PAST e EUC, a fração do COP na camada de 0,00-0,05 m não apresentou sensibilidade às alterações do manejo agrícola (Tabela 1), diferentemente do que geralmente se evidencia em estudos sobre fracionamento da MOS, conforme observado em Souza et al. (2016), Santos et al. (2013); Bayer et al. (2004). Estes autores abordam a fração COP como um indicador mais sensível à qualidade dos sistemas de manejo do solo à curto prazo, contrariamente ao que se observou neste estudo. Todavia, ao se observar os índices L, IC e IMC, os sistemas de uso PAST e LAV-PD se destacam do tratamento EUC. A tabela 1 mostra que, enquanto os tratamentos PAST e LAV-PD apresentam respectivamente, valores de $\mathrm{L}$ de 0,47 e 0,43 , valores de IL de 0,17 e 0,16 e valores de IMC de 20,28 e 21,15, o tratamento EUC apresentou valores muito inferiores de 
Tabela 1 - Valores médios dos teores de carbono orgânico total (COT), do carbono orgânico particulado (COP), do carbono orgânico associado aos minerais (CAM), da relação entre o carbono orgânico particulado e o teor de carbono orgânico total (COP/COT), da relação entre o carbono orgânico associado aos minerais e o teor de carbono orgânico total (CAM/COT), do índice de estoque de carbono (IEC), da labilidade (L), do índice de labilidade (IL) e do índice de manejo do carbono (IMC) de um Latossolo Vermelho submetido a diferentes sistemas de uso.

\begin{tabular}{|c|c|c|c|c|c|c|c|c|c|}
\hline $\begin{array}{c}\text { Sistemas } \\
\text { de Uso }\end{array}$ & \multicolumn{9}{|c|}{-------------Mg m${ }^{-3}$------------- $\quad$---------- \% ---------- } \\
\hline & \multicolumn{9}{|c|}{ Camada de $0,00-0,05 \mathrm{~m}$} \\
\hline LAV-PD & $8,72 \mathrm{a}$ & $2,64 \mathrm{~b}$ & $6,08 \mathrm{ab}$ & 30 & 70 & 1,32 & 0,43 & 0,16 & 21,15 \\
\hline PAST & $7,80 \mathrm{ab}$ & $2,48 \mathrm{~b}$ & $5,33 \mathrm{~b}$ & 32 & 68 & 1,18 & 0,47 & 0,17 & 20,28 \\
\hline EUC & $10,33 \mathrm{a}$ & $1,47 \mathrm{~b}$ & $8,86 \mathrm{a}$ & 14 & 86 & 1,56 & 0,17 & 0,06 & 9,57 \\
\hline \multirow[t]{2}{*}{ MATA } & $6,62 \mathrm{~b}$ & $4,84 \mathrm{a}$ & $1,79 \mathrm{c}$ & 73 & 27 & 1,00 & 2,70 & 1,00 & 100 \\
\hline & \multicolumn{9}{|c|}{ Camada de $0,05-0,10 \mathrm{~m}$} \\
\hline LAV-PD & $6,95 \mathrm{~ns}$ & $0,98 \mathrm{~ns}$ & $5,97 \mathrm{~ns}$ & 14 & 86 & 0,74 & 0,16 & 0,75 & 55,75 \\
\hline PAST & $9,03 \mathrm{~ns}$ & $1,16 \mathrm{~ns}$ & $7,87 \mathrm{~ns}$ & 13 & 87 & 0,96 & 0,15 & 0,68 & 65,04 \\
\hline EUC & $8,23 \mathrm{~ns}$ & $1,25 \mathrm{~ns}$ & $6,98 \mathrm{~ns}$ & 15 & 85 & 0,88 & 0,18 & 0,82 & 72,02 \\
\hline \multirow[t]{2}{*}{ MATA } & $9,38 \mathrm{~ns}$ & $1,68 \mathrm{~ns}$ & $7,70 \mathrm{~ns}$ & 18 & 82 & 1,00 & 0,22 & 1,00 & 100 \\
\hline & \multicolumn{9}{|c|}{ Camada de $0,10-0,20 \mathrm{~m}$} \\
\hline LAV-PD & $6,71 \mathrm{~ns}$ & $1,54 \mathrm{~ns}$ & $5,17 \mathrm{~ns}$ & 23 & 77 & 1,08 & 0,30 & 1,10 & 119,18 \\
\hline PAST & $8,58 \mathrm{~ns}$ & $1,77 \mathrm{~ns}$ & $6,81 \mathrm{~ns}$ & 21 & 79 & 1,38 & 0,26 & 0,96 & 132,97 \\
\hline EUC & $6,66 \mathrm{~ns}$ & $1,84 \mathrm{~ns}$ & $4,82 \mathrm{~ns}$ & 28 & 72 & 1,07 & 0,38 & 1,41 & 151,60 \\
\hline \multirow[t]{2}{*}{ MATA } & $6,20 \mathrm{~ns}$ & $1,32 \mathrm{~ns}$ & $4,88 \mathrm{~ns}$ & 21 & 79 & 1,00 & 0,27 & 1,00 & 100 \\
\hline & \multicolumn{9}{|c|}{ Camada de $0,20-0,40 \mathrm{~m}$} \\
\hline LAV-PD & $6,11 \mathrm{~ns}$ & $1,23 \mathrm{~ns}$ & $4,88 \mathrm{~ns}$ & 20 & 80 & 0,94 & 0,25 & 0,35 & 33,05 \\
\hline PAST & $7,31 \mathrm{~ns}$ & $2,20 \mathrm{~ns}$ & $5,11 \mathrm{~ns}$ & 30 & 70 & 1,12 & 0,43 & 0,60 & 67,53 \\
\hline EUC & $6,70 \mathrm{~ns}$ & $2,23 \mathrm{~ns}$ & $4,46 \mathrm{~ns}$ & 33 & 67 & 1,03 & 0,50 & 0,70 & 71,89 \\
\hline MATA & $6,50 \mathrm{~ns}$ & $2,71 \mathrm{~ns}$ & $3,78 \mathrm{~ns}$ & 42 & 58 & 1,00 & 0,71 & 1,00 & 100 \\
\hline
\end{tabular}

Médias seguidas pela mesma letra minúscula na coluna, dentro de cada camada não diferem entre si pelo teste de Tukey a $5 \%$ de probabilidade. ns: não significativo. LAV-PD: Lavoura com culturas anuais em sistema plantio direto por 5 anos. PAST: pastagem com grama-estrela; EUC: Floresta de eucaliptos com 10 anos de idade; MATA: Mata nativa.

$\mathrm{L}(0,16)$, de IL $(0,06)$ e de IMC $(9,57)$. Isto significa que os tratamentos PAST e LAV-PD, embora apresentando valores muito inferiores à testemunha MATA, são os sistemas de uso que mostram maior potencial para preservação e recuperação do carbono e seus compartimentos no solo em relação ao tratamento EUC, na camada de 0,00-0,05m.O menor valor dos índices L, IL e IMC apresentado pelo tratamento EUC (Tabela 1) deve-se possivelmente ao seu maior conteúdo de CAM em relação ao COT (86\%). Importante ressaltar que a fração CAM apresentou maior sensibilidade em avaliar os diferentes sistemas de uso do solo na camada de 0,00-0,05 m. Isto é, enquanto o tratamento EUC apresentou o maior teor de CAM $\left(8,86 \mathrm{Mg} \mathrm{ha}^{-1}\right)$, o tratamento MATA apresentou o menor teor $\left(1,79 \mathrm{Mg} \mathrm{ha}^{-1}\right)$ e o tratamento LAV-PD não diferiu significativamente do tratamento EUC. O tratamento PAST, apesar de também apresentar menor teor de CAM em relação ao EUC, ainda foi superior ao tratamento MATA na camada de 0,00-0,05 m (Tabela 1). Este resultado deve-se, possivelmente, a qualidade dos resíduos vegetais depositados na superfície do solo dos diferentes sistemas de uso em relação ao ecossistema natural. Os maiores valores de CAM apresentados pelos tratamentos EUC, PAST e LAVPD devem-se, possivelmente a maior recalcitrância molecular dos compostos apresentados pelos tratamentos sob manejo agrícola. Ou seja, no caso da PAST e LAV-PD, as gramíneas que compõem o sistema de uso tendem a possuir maior teor de lignina, o que dificulta o ataque microbiano desta molécula orgânica. Resultados semelhantes foram observados por Ribeiro (2016) e Santos et al. (2014)

Em relação ao tratamento EUC, o elevado teor de CAM deve-se também a qualidade dos resíduos, principalmente por conta do teor de lignina e celulose contido nos compostos orgânicos provenientes da decomposição dos resíduos vegetais. 
Tabela 2 - Valores médios do diâmetro médio geométrico dos agregados (DMG), da densidade do solo (Ds), da macroporosidade (Ma), da microporosidade (Mi) e da porosidade total (Pt) de um Latossolo Vermelho submetido a diferentes sistemas de uso.

\begin{tabular}{|c|c|c|c|c|c|}
\hline Sistemas de Uso & $\begin{array}{c}\mathrm{DMG} \\
\mathrm{mm}\end{array}$ & $\begin{array}{c}\text { Ds } \\
\mathrm{Mg} \mathrm{m}^{-3}\end{array}$ & $\mathrm{Ma}$ & $\begin{array}{c}\mathrm{Mi} \\
---\mathrm{m}^{3} \mathrm{~m}^{-3}-\end{array}$ & $\mathrm{Pt}$ \\
\hline & \multicolumn{5}{|c|}{ Camada de 0,00-0,05 m } \\
\hline LAV-PD & $1,08 \mathrm{~b}$ & $1,48 \mathrm{a}$ & $0,16 \mathrm{~b}$ & $0,33 \mathrm{ab}$ & $0,49 \mathrm{~b}$ \\
\hline PAST & $1,42 \mathrm{ab}$ & $1,51 \mathrm{a}$ & $0,14 \mathrm{~b}$ & $0,38 \mathrm{a}$ & $0,52 \mathrm{ab}$ \\
\hline EUC & $1,82 \mathrm{a}$ & $1,40 \mathrm{a}$ & $0,17 \mathrm{~b}$ & $0,34 \mathrm{ab}$ & $0,52 \mathrm{ab}$ \\
\hline \multirow[t]{2}{*}{ MATA } & $1,79 \mathrm{a}$ & $1,17 \mathrm{~b}$ & $0,27 \mathrm{a}$ & $0,28 \mathrm{~b}$ & $0,56 \mathrm{a}$ \\
\hline & \multicolumn{5}{|c|}{ Camada de 0,05-0,10 m } \\
\hline LAV-PD & $0,97 \mathrm{~b}$ & $1,57 \mathrm{a}$ & $0,15 \mathrm{~b}$ & $0,32 \mathrm{ab}$ & $0,47 \mathrm{~b}$ \\
\hline PAST & $1,20 \mathrm{ab}$ & $1,52 \mathrm{a}$ & $0,15 \mathrm{~b}$ & $0,37 \mathrm{a}$ & $0,53 \mathrm{ab}$ \\
\hline EUC & $1,65 \mathrm{a}$ & $1,49 \mathrm{a}$ & $0,20 \mathrm{~b}$ & $0,33 \mathrm{ab}$ & $0,53 \mathrm{ab}$ \\
\hline \multirow[t]{2}{*}{ MATA } & $1,66 \mathrm{a}$ & $1,28 \mathrm{~b}$ & $0,24 \mathrm{a}$ & $0,27 \mathrm{~b}$ & $0,55 \mathrm{a}$ \\
\hline & \multicolumn{5}{|c|}{ Camada de $0,10-0,20 \mathrm{~m}$} \\
\hline LAV-PD & $1,34 \mathrm{ab}$ & $1,63 \mathrm{a}$ & $0,14 \mathrm{c}$ & $0,33 \mathrm{ab}$ & $0,48 \mathrm{~b}$ \\
\hline PAST & $1,11 \mathrm{~b}$ & $1,54 \mathrm{a}$ & $0,15 \mathrm{c}$ & $0,39 \mathrm{a}$ & $0,57 \mathrm{a}$ \\
\hline EUC & $1,04 \mathrm{~b}$ & $1,50 \mathrm{a}$ & $0,20 \mathrm{~b}$ & $0,32 \mathrm{ab}$ & $0,52 \mathrm{ab}$ \\
\hline \multirow[t]{2}{*}{ MATA } & $1,72 \mathrm{a}$ & $1,23 \mathrm{~b}$ & $0,28 \mathrm{a}$ & $0,29 \mathrm{~b}$ & $0,57 \mathrm{a}$ \\
\hline & \multicolumn{5}{|c|}{ Camada de $0,20-0,40 \mathrm{~m}$} \\
\hline LAV-PD & $1,20 \mathrm{~b}$ & $1,58 \mathrm{a}$ & $0,16 b$ & $0,34 \mathrm{ab}$ & $0,50 \mathrm{~ns}$ \\
\hline PAST & $1,11 \mathrm{~b}$ & $1,46 \mathrm{a}$ & $0,16 \mathrm{~b}$ & $0,35 \mathrm{a}$ & $0,52 \mathrm{~ns}$ \\
\hline EUC & $1,10 \mathrm{~b}$ & $1,57 \mathrm{a}$ & $0,15 \mathrm{~b}$ & $0,35 \mathrm{a}$ & $0,50 \mathrm{~ns}$ \\
\hline MATA & $1,74 \mathrm{a}$ & $1,26 \mathrm{~b}$ & $0,23 \mathrm{a}$ & $0,30 \mathrm{~b}$ & $0,54 \mathrm{~ns}$ \\
\hline
\end{tabular}

Médias seguidas pela mesma letra minúscula na coluna, dentro de cada camada não diferem entre si pelo teste de Tukey a 5\% de probabilidade. ns: não significativo. LAV-PD: Lavoura com culturas anuais em sistema plantio direto por 5 anos. PAST: pastagem com grama-estrela; EUC: Floresta de eucaliptos com 10 anos de idade; MATA: Mata nativa.

Barreto et al. (2014) também observaram em florestas de eucalipto que, com o aumento da idade, maiores eram os teores de celulose e lignina, justamente os compostos que influenciam na velocidade de decomposição dos resíduos, os quais limitaram o acesso dos microrganismos ao substrato, proporcionando um maior nível de recalcitrância à serapilheira.

Por outro lado, no ambiente de mata nativa, naturalmente, há uma maior diversidade de espécies vegetais, com deposição de resíduos em quantidades e qualidades diferentes, onde ocorre maior ciclagem, pela maior presença de outros organismos como ácaros, colembolos, fungos, bactérias, etc. (BRADY; WEILL, 2013). Portanto, a menor relação CAM/COT do tratamento MATA evidencia a presença de compostos mais alifáticos nos resíduos depositados na camada superficial do solo e, consequentemente, mais favoráveis ao ataque biológico.

Nas camadas subjacentes a 0,00-0,05 m, em geral, se observou similaridade entre os teores de COT, COP e CAM. Todavia, somente na camada de 0,10-
0,20 $\mathrm{m}$ os valores de COP/COT, L e IMC dos tratamentos sob manejo agrícola foram iguais ou superiores ao tratamento MATA (Tabela 1). Este resultado pode ser devido ao efeito positivo da concentração de raízes das gramíneas e do eucalipto especificamente nesta camada do solo (Tabela 1). De acordo com Jackson et al. (1996), a média global da profundidade das raízes das gramíneas nos diferentes biomas mostra que cerca de $50 \%$ das raízes se localizam nos primeiros 0,20 $\mathrm{m}$ do solo. Witschoreck et al. (2003), avaliando a biomassa radicular de eucaliptos com 10 anos de idade, observaram em torno de $60 \%$ de raízes finas se concentraram até $0,20 \mathrm{~m}$ de profundidade.

Quando se analisa os atributos físicos do solo nas diferentes camadas analisadas (Tabela 2), o tratamento MATA se destaca dos demais tratamentos sob manejo agrícola. Importante ressaltar que, conforme mencionado por Braida e Reichert (2014), mesmo quando áreas sob uso agrícola apresentam maior incremento no teor de COT em relação às áreas naturais, como ocorreu no presente estudo na camada de $0,00-0,05 \mathrm{~m}$, estas não necessariamente apresentam 
melhorias nos atributos físicos do solo, pois sob uso agrícola, o solo é submetido à aplicação de cargas superficiais que superam em magnitude, frequência e abrangência àquelas aplicadas em condições naturais. Isto de fato, foi observado nos resultados de agregação, Ds e porosidade do solo em estudo, onde o tratamento MATA apesar de apresentar o menor teor de COT na camada de 0,00-0,05 m (Tabela 1), esta apresentou um dos maiores valores de DMG, menor valor de Ds e maior Ma e Pt nesta camada em relação aos demais sistemas de uso, principalmente em relação aos tratamentos LAV-PD e PAST (Tabela 2).

Em relação ao DMG, se observou que até a profundidade de 0,00-0,10 m, os tratamentos MATA e EUC se destacaram do tratamento LAV-PD. Enquanto que na camada de 0,00-0,05 m, os valores de DMG da MATA e EUC foram, respectivamente, 66 e $69 \%$ superiores ao tratamento LAV-PD, na camada de 0,05$0,10 \mathrm{~m}$, os valores foram, respectivamente, 71 e $70 \%$ superiores (Tabela 2). Luciano et al. (2010), também observaram menores valores de DMG em áreas sob plantio direto há 2 anos quando comparados à uma área de mata, todavia, em áreas sob plantio direto há 5 anos, os autores observaram valores de DMG muito próximos as da área de mata nativa. Isto se deve pelo fato de que, em áreas de lavoura com cultivos anuais, o crescimento de raízes de plantas é sazonal, concentrando-se quase que exclusivamente nos períodos de crescimento vegetativo das culturas (BRAIDA; REICHERT, 2014), necessitando, portanto, um período maior de implantação do sistema plantio direto para que o efeito da cobertura permanente do solo se traduza em benefícios aos atributos físicos do solo. Logo, somado ao tempo de implantação do tratamento LAV-PD ser recente, a renovação e a diversidade radicular, a distribuição uniforme de exudados no solo pelas plantas e a grande diversidade de organismos do solo em áreas naturais, fazem com que estas sejam áreas que apresentem maior estabilidade de agregados em água em relação a áreas agrícolas sob manejo conservacionista.

As diferenças do DMG da área de EUC em relação ao LAV-PD devem-se ao período de implantação da área florestal, isto é, o não revolvimento do solo por 10 anos e a presença de um sistema radicular mais agressivo acarretou, possivelmente, a maior agregação nas primeiras camadas do solo. Resultados semelhantes foram observados por Loss et al. (2014) e Wendling et al. (2012).

A partir da camada de 0,10-0,20 m, a área de MATA apresentou o maior DMG em relação às demais áreas estudadas. Enquanto que na camada de 0,10-0,20 $\mathrm{m}$ os valores de $\mathrm{DMG}$ da mata nativa foram superiores em $65 \%, 55 \%$ e $28 \%$ em relação à área com EUC, PAST e LAV-PD, respectivamente, na camada de 0,20$0,40 \mathrm{~m}$ os valores foram $58 \%, 57 \%$ e $45 \%$ superiores, respectivamente (Tabela 2). Isto evidencia o maior estado de organização do solo sob sistema natural, inclusive em superfície, mesmo quando apresenta menor teor de COT. De acordo com Braida e Reichert et al. (2014) a maior diversidade vegetal em ecossitemas não antropizados, sempre implica em maior diversidade de outros organismos vivos, os quais contribuem para o desenvolvimento de uma estrutura mais porosa e menos densa no solo. Esta condição física se observa em todas as camadas do solo sob MATA, onde este tratamento apresentou a menor Ds e maior $\mathrm{Ma}$ em relação aos demais tratamentos (Tabela 2).

Importante ressaltar que os valores de Ds $>1,40 \mathrm{Mg} \mathrm{m} \mathrm{m}^{-3}$, considerados restritivos para a maioria das culturas agrícolas em solos de textura argilosa (REICHERT et al., 2003; REICHERT et al., 2009; GIRARDELO et al., 2011), foram observados em todos os demais sistemas de uso, nas diferentes camadas de solo. Este resultado evidencia, possivelmente, a persistência do efeito do trafego de máquinas durante a implantação e manejo das áreas de EUC, PAST e LAV-PD. De acordo com Reichert et al. (2007), a profundidade de compactação é variável com as especificações da máquina e seu rodado, condições do solo, número de vezes em que o solo é trafegado e com seu histórico de pressões, além do manejo (se pastagem, floresta ou agricultura). $\mathrm{Na}$ agricultura, a profundidade de compactação é variável graças, principalmente, ao manejo do solo: se plantio direto ou convencional, se escarificação ou subsolagem.

De forma geral, ecossistemas naturais, justamente por não terem sofrido alterações pelas práticas de manejo, tendem a apresentar maior percentual de porosidade total e macroporosidade em relação aos solos cultivados (MARASCA et al., 2013). Este comportamento em relação a Ma principalmente, foi observado no tratamento MATA, que diferiu significativamente dos demais sistemas de uso, em todas as camadas do solo, apresentando valores superiores a $0,20 \mathrm{~m}^{3} \mathrm{~m}^{-3}$ (Tabela 2). Segundo Viana et al. (2011), a mata por ser um sistema mais equilibrado em todos os seus seguimentos apresenta maior percentual de porosidade em relação ao outros sistemas de uso e manejo que recebam maior revolvimento. Contudo, os sistemas de uso EUC, PAST e LAV-PD, apesar de apresentarem períodos distintos de implantação, já apresentam efeitos positivos da presença de cobertura permanente do solo e do cessar do revolvimento, exemplificadas pelos valores de $\mathrm{Ma}$, que foram, em todas as camadas analisadas, superiores a $0,10 \mathrm{~m}^{3} \mathrm{~m}^{-3}$ (Tabela 2), considerado um valor adequado ao desenvolvimento de plantas (REICHERT et al., 2003; REICHERT et al., 2009; GIRARDELO et al., 2011). Segundo Calonego et al. (2011), o desenvolvimento radicular está diretamente relacionado à condição estrutural do solo, pois o caminho natural de crescimento das raízes é predominantemente pelos macroporos. Portanto, deve-se ter o cuidado ao manejar áreas com intenso pisoteio animal e/ou elevado tráfego de máquinas, pois estas tendem a aumentar a densidade e reduzir a porosidade do solo, favorecendo a sua degradação (BERGAMIN et al., 
2010; COLLARES et al., 2011). Por outro lado, diferentes estudos vêm mostrando que em longo prazo, sistemas conservacionistas, como o plantio direto, melhoram os atributos físicos do solo, inclusive se aproximando de valores encontrados em ecossistemas naturais, conforme mostrado por Souza et al. (2016) e Reichert et al. (2016).

Importante ressaltar que, apesar dos valores elevados do IMC dos tratamentos LAV-PD e PAST em relação ao tratamento EUC na camada de 0,00-0,05 m (Tabela 1), isto não se traduziu em melhores resultados de DMG, Ds e Ma entre estes tratamentos (Tabela 2), evidenciando que o teor de carbono e de suas diferentes frações ainda devem ser incipientes ao ponto de se traduzir em melhorias no sistema solo sob o cultivo destas espécies.

\section{CONCLUSÕES}

O estudo mostrou a importância de se realizar avaliações quali-quantitativas do teor de carbono do solo (COT, COP e CAM), no intuito de se avaliar de uma forma mais precisa a contribuição dos sistemas de uso sobre a dinâmica do carbono no ambiente e nos atributos físicos do solo.

O carbono orgânico associado aos minerais apresenta maior sensibilidade entre os tratamentos analisados quando comparado ao carbono orgânico particulado, principalmente na camada de 0,00-0,05 m.

Os maiores índices de manejo do carbono dos tratamentos LAV-PD e PAST em relação ao tratamento EUC não culminaram em melhores resultados dos atributos físicos destes tratamentos, mostrando que este índice deve sempre ser avaliado juntamente com determinações de outros atributos do solo, para melhor entender a contribuição do carbono nos diferentes sistemas de uso do solo.

O tratamento MATA mostrou-se ser o sistema mais equilibrado, considerando todas as camadas de solo avaliadas, pois apresenta a maior estabilidade dos agregados, menor densidade do solo e maior percentual de porosidade total e macroporos em relação aos demais tratamentos, mesmo apresentando um menor teor de COT na camada superficial de 0,05 $\mathrm{m}$.

O tratamento EUC, apesar de apresentar DMG semelhante ao tratamento MATA até a camada de $0,10 \mathrm{~m}$, ainda é um sistema de uso que precisa de um maior tempo de consolidação para promover melhorias de Ds e porosidade no solo.

\section{REFERÊNCIAS}

ALVARES, C.A.; STAPE, J. L.; SENTELHAS, P.C.; GONÇALVES, J.L.M.; SPAROVEK, G. Kooppen's climate classification map for Brazil. Meteorologische Zeitschrift, v. 22, n. 6, p. 711-728, 2013.
AMORIM, F.F. Agregação e estabilidade da matéria orgânica em sistemas conservacionistas de manejo do solo. 2016. 80 p. Dissertação (Mestrado em Ciência do Solo) - Faculdade de Agronomia, Universidade Federal do Rio Grande do Sul, 2016.

BARRETO, P.A.B.; GAMA-RODRIGUES, E.F.D.; GAMA-RODRIGUES, A.C. Carbono das frações da matéria orgânica em solos sob plantações de eucalipto de diferentes idades. Scientia Forestalis, v. 42, n. 104, p. 581-590, 2014.

BAYER, C.; MARTIN-NETO, L.; MIELNICZUK, J.; PAVINATO, A. Armazenamento de carbono em frações lábeis da matéria orgânica de um Latossolo Vermelho sob plantio direto. Pesquisa Agropecuária Brasileira, v. 39, n. 7, p. 677-683, 2004.

BERGAMIN, A.C.; VITORINO, A.C.T.; FRANSCHINI, J.C.; SOUZA, C.M.A.; SOUZA, F.R. Compactação em um Latossolo vermelho distroférrico e suas relações com o crescimento radicular do milho. Revista Brasileira de Ciência do Solo, v. 34, n. 1, p. 681-691, 2010.

BLAIR, G.J.; LEFROY, R. D. B.; LISE, L. Soil carbon fractions based on their degree of oxidation and the development of a carbon management index for agricultural systems. Australian Journal of Agricultural Research, v. 46, n. 7, p. 1459-1466, 1995.

BALOTA, E.L. Manejo e qualidade biológica do solo. Londrina: Mecenas, 2017.

BRADY, N.C.; WEIL, R.R. Elementos da natureza e propriedades dos solos. Porto Alegre: Bookmann Editora LTDA, 2013.

BRAIDA, J.A.; REICHERT, J.M. Matéria orgânica e comportamento mecânico para fins de manejo de solo. In: LEITE, L.F.C.; MACIEL, G.A.; ARAÚJO, A.S.F. Agricultura Conservacionista no Brasil. Brasilia, DF: Embrapa, 2014. cap. 3, p. 309-361.

CALONEGO, J.C.; GOMES, T.C.; SANTOS, C.H. dos; TIRITAN, C.S. Desenvolvimento de plantas de cobertura em solo compactado. Bioscience Journal, v. 27, n.2, p. 289-296, 2011.

CAMBARDELLA, C,A.; ELLIOT, E.T. Particulate soil organic-matter changes across a grassland cultivation sequence. Soil Science Society of America Journal, v. 56, n. 3, p. 777-783, 1992.

CAMPOS, B.C.; AMADO,T.J.C.; BAYER, C.; NICOLOSO, R. S.; FIORIN,J.E. Carbon stock and its compartments in a subtropical oxisol under long-term tillage and crop rotation systems. Revista Brasileira de Ciência do Solo, v. 35, n. 3, p. 805-817, 2011. 
CARPENEDO, V.; MIELNICZUK, J. Estado de agregação e qualidade de agregados de Latossolos Roxos, submetidos a diferentes sistemas de manejo. Revista Brasileira de Ciência do Solo, v. 14, p. 99-105, 1990.

COLLARES, G.L.; REINERT, D.J.; REICHERT, J.M.; KAISER, D.R. Compactação superficial de Latossolos sob integração lavoura - pecuária de leite no noroeste do Rio Grande do Sul. Ciência Rural, v. 41, n. 2, p. 246-250, 2011.

CONCEIÇÃO, P.C.; BAYER, C.; DIEKOW, J.; SANTOS, D.C.D. Fracionamento físico da matéria orgânica e índice de manejo de carbono de um Argissolo submetido a sistemas conservacionistas de manejo. Ciência rural, v. 44, n. 5, p. 794-800, 2014.

CONCEIÇÃO, P. C. Agregação e proteção física da matéria orgânica em dois solos do sul do Brasil. 2006. 138 p. Tese (Doutorado em Ciência do Solo) - Faculdade de Agronomia, Universidade Federal do Rio Grande do Sul, 2006.

COSER, T.R.; RAMOS, M.L.G.; FIGUEIREDO, C.C.D.; CARVALHO, A.M.D.; CAVALCANTE, E.; MOREIRA, M.K.D.R.; ARAUJO, P.S.; OLIVEIRA, S.A.D. Soil microbiological properties and available nitrogen for corn in monoculture and intercropped with forage. Pesquisa Agropecuária Brasileira, v. 51, n.9, p. 1660-1667, 2016.

CUNHA, E.Q.; STONE, L.F.; FERREIRA, E.P.B.; DIDONET, A.D.; MOREIRA, J.A.A. Atributos físicos, químicos e biológicos de solo sob produção orgânica impactados por sistemas de cultivo. Revista Brasileira de Engenharia Agricola e Ambiental, v. 16, n. 1, p.56-63, 2012.

DICK, D.P.; NOVOTNY, E.H.; DIECKOW, J.; BAYER, C. Química da matéria orgânica do solo. In: FREITAS MELO, V.; ALLEONI, L.R.F. Quimica e mineralogia do solo. Viçosa, MG: Sociedade Brasileira de Ciência do Solo, 2009. Parte II, p. 1-68.

DIECKOW, J.; MARTIN-NETO, L.; MILORI, D. M.B.P.; CONCEIÇÃO, P.C.; BAYER, C.; MIELNICAZUK, J. Sistemas de preparo do solo e características espectroscópicas da matéria orgânica em ambientes tropicais e subtropicais brasileiros. Boletim de Pesquisa e Desenvolvimento, n. 12. Embrapa Instrumentação Agropecuária, 2005.

DONAGEMA, G.K.; CAMPOS, D.V.B. de; CALDERANO, S.B.; TEIXEIRA, W.G.; VIANA, J.H.M. Manual de métodos de análise de solo. Rio de Janeiro: Embrapa Solos, 2011. 230 p.
EMBRAPA. Centro Nacional de Pesquisa de Solos. Sistema Brasileiro de Classificação de Solos. 3 ed. Brasilia, DF.: Embrapa Solos, 2013.

ENSINAS, S.C.; SERRA, A.P.; MARCHETTI, M.E.; DA SILVA, E.F.; FERREIRA DO PRADO, E.A.; LOURENTE, E.R.P.; ALTOMAR, P.H.; POTRICH, D.C; MARTINEZ, M.A.; CONRAD, V.A.; JESUS, M.V.; EL KADRI, T.C. Cover crops affect on soil organic matter fractions under no till system. Australian Journal of Crop Science, v. 10, n. 4, p. 503-512, 2016.

FACCIN, F.C.; MARCHETTI, M.E.; SERRA, A.P.; ENSINAS, S.C. Frações granulométricas da matéria orgânica do solo em consórcio de milho safrinha com capim-marandu sob fontes de nitrogênio. Pesquisa Agropecuária Brasileira, v. 51, n. 12, p. 2000-2009, 2017.

FONTE, S.J.; NESPER, M.; HEGGLIN, D.; VELÁSQUEZ, J.E.; RAMIREZ, B.; RAO, I. M.; BERNASCONI, S.M.; BÜNEMANN, E.K.; FROSSARD, E.; OBERSON, A. Pasture degradation impacts soil phosphorus storage via changes to aggregate-associated soil organic matter in highly weathered tropical soils. Soil Biology and Biochemistry, v. 68, n. 1, p. 150-157, 2014.

GIRARDELLO, V.C.; AMADO, T.J.C.; NICOLOSO, R. da S.; HÖRBE, T. de A.N.; FERREIRA, A. de O.; TABALDI, F.M.; LANZANOVA, M.E. Alterações nos atributos físicos de um Latossolo Vermelho sob plantio direto induzidas por diferentes tipos de escarificadores e o rendimento da soja. Revista Brasileira de Ciência do Solo, v.35, p.2115-2126, 2011.

JACKSON, R.B.; CANADELL, J.; EHLERINGER, J.R.; MOONEY, H.A.; SALA, O.E.; SCHULZE, E.D. A global analysis of root distributions for terrestrial biomes. Oceologia, v.108, n. 3, p.389-411, 1996.

KUNDE, R.J.; LIMA, C.L.R., DOS ANJOS SILVA, S.D.; PILLON, C.N. Frações físicas da matéria orgânica em Latossolo cultivado com cana-de-açúcar no Rio Grande do Sul. Pesquisa Agropecuária Brasileira, v. 51, n. 9, p. 1520-1528 2016.

LAL, R. Restoring soil quality to mitigate soil degradation. Sustainability, v. 7, n. 5, p. 5875-5895, 2015.

LEAL, O.D.A.; CASTILHOS, R.M.V.; PINTO, L.F.S.; PAULETTO, E.A.; LEMES, E.S.; KUNDE, R.J. Initial Recovery of Organic Matter of a Grass-Covered Constructed Soil after Coal Mining. Revista Brasileira de Ciência do Solo, v. 40, n. 1, p. 1-16, 2016. 
LIMA, A.M.N.; SILVA, I.D.; NEVES, J.C.L.; NOVAIS, R.D.; BARROS, N.D.; MENDONÇA, E.D.S.; LEITE, F.P. Frações da matéria orgânica do solo após três décadas de cultivo de eucalipto no Vale do Rio Doce-MG. Revista Brasileira de Ciência do Solo, v. 32, n. 2, p. 1053-1063, 2008.

LOSS, A.; RIBEIRO, E.C.; PEREIRA, M.G.; COSTA, E. M. Atributos físicos e químicos do solo em sistemas de consórcio e sucessão de lavoura, pastagem e silvipastoril em Santa Teresa, ES. Bioscience Journal, v. 30, n. 5, p. 1347-1357, 2014.

LOSS, A.; PEREIRA, M.G.; SCHULTZ, N.; CUNHA DOS ANJOS, L.H.; RIBEIRO DA SILVA, E.M. Frações orgânicas e índice de manejo de carbono do solo em diferentes sistemas de produção orgânica. Idesia (Arica), v. 29, n. 2, p. 11-19, 2011.

LUCIANO, R.V.; BERTOL, I.; BARBOSA, F.T.; KURTZ, C.; FAYAD, J.A. Propriedades físicas e carbono orgânico do solo sob plantio direto comparados à mata natural, num Cambissolo Háplico. Revista de Ciências Agroveterinárias, v. 9, n.1, p. 9-19. 2014.

MARASCA, I.; GONÇALVES, F.C.; MORAES, M.H.; BALLARIN, A.W.; GUERRA, S.P.S.; LANÇAS, K.P. Propriedades físicas de um Nitossolo Vermelho em função dos sistemas de uso e manejo. Revista Brasileira de Engenharia Agricola e Ambiental, v. 17, n. 11, p. 11601166, 2013.

MENDHAM, D.S.; HEAGNEY, E.C.; CORBEELS, M.; O'CONNELL, A.M.; GROVE, T.S.; MCMURTRIE, R.E. Soil particulate organic matter effects on nitrogen availability after afforestation with Eucalyptus globulus. Soil Biology and Biochemistry, v. 36, n. 7, p. 1067-1074, 2004.

REICHERT, J. M.; DA ROSA, V.T.; VOGELMANN, E.S.; DA ROSA, D.P.; HORN, R.; REINERT, D.J.; SATTLER, A.; DENARDIN, J.E.. Conceptual framework for capacity and intensity physical soil properties affected by short and long-term (14 years) continuous no-tillage and controlled traffic. Soil \&Tillage Research, v. 158, p. 123-136, 2016.

REICHERT, J.M.; KAISER, D.R.; REINERT, D.J.; RIQUELME, U.F.B. Variação temporal de propriedades físicas do solo e crescimento radicular de feijoeiro em quatro sistemas de manejo. Pesquisa Agropecuária Brasileira, v. 44, n. 3, p. 310-319, 2009.

REICHERT, J.M.; SUZUKI, L.E.A.S.; REINERT, D. J. Compactação do solo em sistemas agropecuários e florestais: identificação, efeitos, limites críticos e mitigação. In: CERRETA, C.A.; SILVA, L.S.; REICHERT. J.M. Tópicos em ciência do solo, 2007. Viçosa: MG. p. 49-134.
REICHERT, J.M.; REINERT, D.J.; BRAIDA, J.A. Qualidade dos solos e sustentabilidade dos sistemas agrícolas. Ciência e Ambiente, v.27, p.29-48, 2003.

REIS, D.A.; LIMA, C.L.R.; BAMBERG, A.L. Qualidade física e frações da matéria orgânica de um Planossolo sob sistema plantio direto. Pesquisa Agropecuária Brasileira, v. 51, n.9, p. 1623-1632, 2016.

RIBEIRO, L.R.P. Efeitos de plantas de cobertura e da adubação nitrogenada nas fraçôes da matéria orgânica do solo e na produtividade do milho. 53 p. Dissertação (Mestrado em Agronomia) - Faculdade de Agronomia e Medicina Veterinária - Universidade de Brasília, 2016.

ROSSETTI, K.V.; CENTURION, J.F. Estoque de carbono e atributos físicos de um Latossolo em cronossequência sob diferentes manejos. Revista Brasileira de Engenharia Agricola e Ambiental, v. 19, n. 3, p. 252-258, 2015.

SALTON, J.C.; MIELNICZUK, J.; BAYER, C.; FABRÍCIO, A.C.; MACEDO, M.C.M.; BROCH, D.L. Teor e dinâmica do carbono no solo em sistemas de integração lavoura-pecuária. Pesquisa Agropecuária Brasileira, v. 46, n. 10, p. 1349-1356, 2012.

SANTOS, I.L.D.; CAIXETA, C.F.; SOUSA, A.A.T.C.D.; FIGUEIREDO, C. C., RAMOS, M. L. G.; CARVALHO, A.M.D. Cover plants and mineral nitrogen: effects on organic matter fractions in an oxisol under no-tillage in the cerrado. Revista Brasileira de Ciência do Solo, v.38, n.6, p. 1874-1881, 2014.

SANTOS, D.C.; OLIVEIRA FARIAS, M.; LIMA, C.L.R.; KUNDEI, R.J.; PILLON, C.N.; FLORES, C.A. Fracionamento químico e físico da matéria orgânica de um Argissolo Vermelho sob diferentes sistemas de uso. Ciência Rural, v. 43, n. 5, 2013.

SILVA, F.A.S.; AZEVEDO, C.A.V. Principal ComponentsAnalysis in the Software AssistatStatisticalAttendance. In: WORLD CONGRESS ON COMPUTERS IN AGRICULTURE, 7, Reno-NVUSA: American Society of Agricultural and Biological Engineers, 2009.

SOUZA, E.D.; CARNEIRO, M.A.C.; BARBOSA PAULINO, H.; RIBEIRO, D.O.; BAYER, C.; ROTTA, L.R. Matéria orgânica e agregação do solo após conversão de "campos de murundus" em sistema plantio direto. Pesquisa Agropecuária Brasileira, v. 51, n.9, p.1194-1202, 2016. 
VIANA, E.T.; BATISTA, M.A.; TORMENA, C.A.; COSTA, A.C.C.; TAKEYSHI, T.I.; Atributos físicos e carbono orgânico em Latossolo vermelho sob diferentes sistemas de uso e manejo. Revista Brasileira de Ciência do Solo, v. 35, n. 1, p.2105-2114, 2011.

WENDLING, B.; VINHAL-FREITAS, I.C.; OLIVEIRA, R.C.; BABATA, M.M.; BORGES, E.N. Densidade, agregação e porosidade do solo em áreas de conversão do cerrado em floresta de pinus, pastagem e plantio direto. Bioscience Journal, v. 28, n. 1, p. 256-265, 2012.
WITSCHORECK, R.; SCHUMACHER, M.V.; CALDEIRA, M.V. W. Estimativa da biomassa e do comprimento de raízes finas em Eucalyptusurophylla S.T. Blake no município de Santa Maria-RS. Revista Árvore, v. 27, n. 2, p. 177-183, 2003.

YEOMANS, J.C.; BREMMER, J.M. A rapid and precise method for routine determination of organic carbon in soil. Journal Communications, Soil Science and Plant Analysis, v. 19, n. 13, p. 1467-1476, 1988. 